Proyecciones Journal of Mathematics

Vol. 36, No 4, pp. 769-778, December 2017.

Universidad Católica del Norte

Antofagasta - Chile

\title{
Función de Dulac en la Familia Loud
}

\author{
Nicole Carrasco Vidal \\ Universidad de Concepción, Chile \\ Received: August 2017. Accepted : October 2017
}

\begin{abstract}
En este trabajo consideramos la familia Loud. Estudiamos la función de Dulac en un caso particular de esta familia. Calculamos el primer término del desarrollo de Dulac de esta función y determinamos que este desarrollo no tiene términos con logaritmos.
\end{abstract}

Keywords:

2010 MSC: 34C07; 34E05. 


\section{Introducción}

En este trabajo consideramos la familia Loud

$$
E_{\mu}:\left\{\begin{array}{l}
\frac{d x}{d t}=-y+B x y \\
\frac{d y}{d t}=x+D x^{2}+F y^{2}
\end{array}\right.
$$

donde $\mu=(B, D, F) \in \mathbf{R}^{3}$. La transformación $(x, y, t) \mapsto(x,-y,-t)$ preserva la ecuación $E_{\mu}$, de modo que el origen es un centro, es decir, es una singularidad rodeada por órbitas cerradas. La región formada por tales órbitas cerradas se llama anillo periódico. La frontera del anillo periódico tiene 2 componentes conexas, una es el centro mismo y la otra es un policiclo $P_{\mu}$ de la extensión natural, $\bar{E} \mu$, de $E \mu$ a la esfera de Poincaré; esto es, $P_{\mu}$ es una unión conexa de singularidades y órbitas regulares de $\bar{E} \mu$.

En este artículo vamos a considerar la siguiente subfamilia de $E_{\mu}$ :

$$
E_{F}:\left\{\begin{array}{l}
\frac{d x}{d t}=-y+x y \\
\frac{d y}{d t}=x+F y^{2}
\end{array}\right.
$$

con $F \in]-1 / 2,0[$.

La ecuación Loud posee (ver [4], por ejemplo) una integral primera de Darboux dada por

$$
I(x, y)=(1-x)^{-2 F}\left(\frac{y^{2}}{2}+\frac{1-x}{1-2 F}+\frac{1}{2 F}\right) .
$$

Así, la recta $\{x=1\}$ y la parábola $\left\{\frac{y^{2}}{2}+\frac{1-x}{1-2 F}+\frac{1}{2 F}=0\right\}$ son curvas invariantes de la ecuación $E_{F}$ y se intersectan en las sillas hiperbólicas $\left(1, \sqrt{\frac{1}{-F}}\right)$, $\left(1,-\sqrt{\frac{1}{-F}}\right)$ formando el policiclo $P_{F}$ antes mencionado. Este policiclo está recorrido en el sentido como se indica en la Figura 1. 


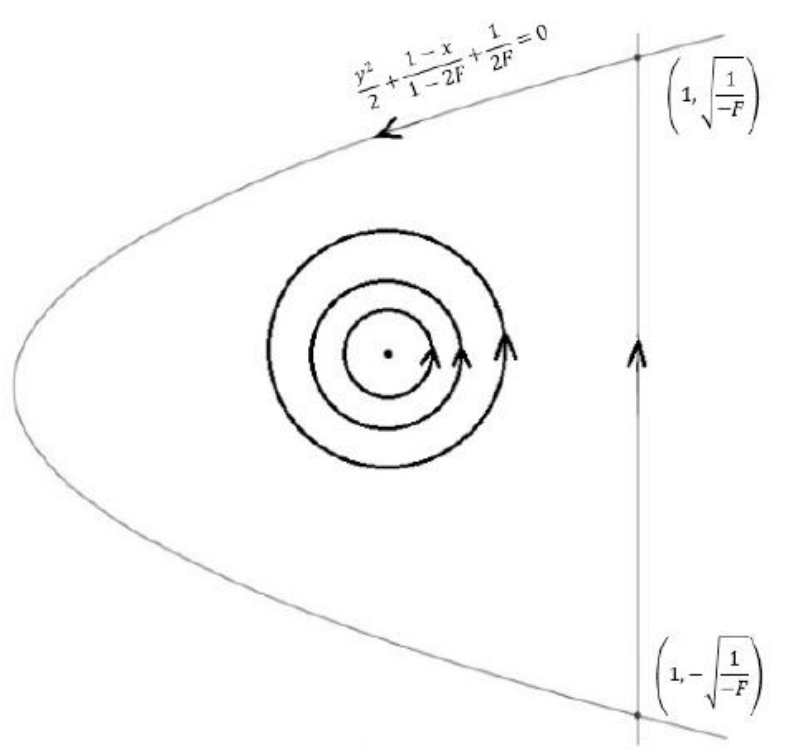

Figure 1: $P_{F}$

Los segmentos $\Sigma=\{y=0, x \approx 1\}$ y $\Pi=\left\{y=0, x \approx \frac{1}{2 F}\right\}$ intersectan transversalmente a $P_{F}$ en los puntos $(1,0)$ y en el vértice $\left(\frac{1}{2 F}, 0\right)$ de la parábola, respectivamente. Parametrizando $\Sigma$ y $\Pi$ respectivamente por las funciones $\sigma:]-\delta, \delta\left[\rightarrow \mathbf{R}^{2}, s \mapsto \sigma(s)=(1-s, 0)\right.$ y $\left.\pi:\right]-\epsilon, \epsilon\left[\rightarrow \mathbf{R}^{2}\right.$, $s \mapsto \pi(s)=\left(\frac{1}{2 F}+s, 0\right)$, para $\delta>0$ y $\epsilon>0$ suficientemente pequeños, la semiórbita positiva de $E_{F}$ que pasa por un punto $\sigma(s)$, con $0<s<\delta$, intersecta a $\Pi$, una primera vez, en $\pi(R(s))$. Se tiene asídefinida la función pasaje de esquina $R:] 0, \delta[\rightarrow] 0, \epsilon[, s \mapsto R(s)$.

De [2] (ver también [5]), sabemos que estas funciones tienen un desarrollo asintótico de Dulac, es decir, para cada $n \in \mathbf{N}$,

$$
R(s)=a s^{\lambda_{0}}+\sum_{k=1}^{n} s^{\lambda_{k}} P_{k}(\log s)+s^{\lambda_{n}} r_{n}(s),
$$

donde $a>0$, la sucesión $\left\{\lambda_{k}\right\}_{k \in \mathbf{N}_{0}}$ es una sucesión estrictamente creciente de números reales positivos, $P_{k}$ es un polinomio real para cada $k \in \mathbf{N}$, y la función $r$ verifica $\lim _{s \rightarrow 0^{+}} r_{n}(s)=0$.

Demostraremos en este trabajo que, en nuestro caso, el desarrollo asintótico de la función $R$ no tiene términos con logaritmos y calcularemos la parte 
principal de $R$, esto es, el primer término no nulo de dicho desarrollo. Más precisamente, demostraremos el siguiente teorema.

La función pasaje de esquina $R$ posee un desarrollo asintótico de Dulac sin términos con logaritmos y verifica la igualdad

$$
R(s ; F)=\left(\frac{1-2 F}{-2 F}\right)^{1+2 F} s^{-2 F}(1+r(s ; F)),
$$

donde la función $r$ está definida y es analítica en $] 0, \delta[\times]-1 / 2,0[$, para

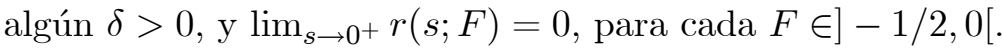

Además del interés intrínseco, el estudio de la función pasaje de esquina es importante en la investigación de bifurcaciones de puntos críticos de la función período (períodos críticos) asociada al anillo periódico. En [1], Chicone conjeturó que si un sistema cuadrático tiene un centro con una función período no monótona entonces, por una transformación afín y un reescalamiento en el tiempo, el sistema puede ser llevado a la forma normal Loud. Por otra parte, en [4], los autores describieron el diagrama de bifurcación de los períodos críticos, dejando conjeturadas algunas líneas, entre ellas el segmento $\{B=1, D=0, F \in]-1 / 2,0[\}$. La función período se escribe como una suma finita de tiempos locales: a lo largo de los lados del policiclo y a lo largo de los sectores sillas, compuestos por funciones de Poincaré y funciones pasajes de esquina. Para completar el diagrama de bifurcación, se estudia la existencia de un desarrollo asintótico de la función período. Para ello se estudia el desarrollo asintótico de cada función involucrada en la definición de la función período, entre ellas las funciones pasajes de esquina.

\section{Demostración del Teorema A}

Para demostrar el teorema A, consideraremos dos puntos adicionales $p_{1}, q_{1} \in$ $P_{F}$ de manera conveniente, con $p_{1} \in\{x=1, y>0\}$ y $q_{1} \in\{x=0, y>0\}$, y dos secciones transversales a $P_{F}$ tales que $\left.\sigma_{1}:\right]-\delta_{1}, \delta_{1}\left[\rightarrow \mathbf{R}^{2}, u \mapsto \sigma_{1}(u)\right.$ у $\left.\pi_{1}:\right]-\epsilon_{1}, \epsilon_{1}\left[\rightarrow \mathbf{R}^{2}, v \mapsto \pi_{1}(v)\right.$ con $\sigma_{1}(0)=p_{1}$ y $\pi_{1}(0)=q_{1}$. Si $\Sigma_{1}$ y $\Pi_{1}$ son las imágenes de $\sigma_{1}$ y $\pi_{1}$ respectivamente, la semiórbita de $E_{F}$ que pasa por un punto $\sigma_{1}(s)$, para $s>0$, intersecta una primera vez a la transversal $\Pi_{1}$ en un punto $\pi_{1}(d(s))$ y la semiórbita positiva que pasa por un punto $\pi_{1}(v)$, con $0<v<\epsilon_{1}$, intersecta una primera vez a la transversal $\Pi$ (introducida en la sección anterior) en $\pi\left(f_{2}(v)\right)$. Se tienen asídefinidas las funciones $d:] 0, \delta_{1}[\rightarrow] 0, \epsilon_{1}\left[, u \mapsto d(u)\right.$ y $\left.f_{2}:\right] 0, \epsilon_{1}[\rightarrow] 0, \epsilon\left[, v \mapsto f_{2}(v)\right.$, lla- 
madas respectivamente función de Dulac y, función de Poincaré entre $\Pi_{1}$ y $\Pi$. Análogamente se define la función $\left.f_{1}:\right] 0, \delta[\rightarrow] 0, \delta_{1}$ [ llamada función de Poincaré entre $\Sigma$ y $\Sigma_{1}$.

Así, la función pasaje de esquina $R$ está dada por la composición

$$
R=f_{2} \circ d \circ f_{1} .
$$

Se sabe que las funciones de Poincaré son difeomorfismos analíticos de la misma clase de diferenciabilidad que de la ecuación diferencial inicial. En lo que sigue, calcularemos el primer término no nulo del desarrollo asintótico de las funciones $f_{1}, f_{2}$ y $d$. En el caso de las funciones $f_{1}$ y $f_{2}$ este término corresponde a sus respectivas derivadas en cero.

\subsection{Estudio de la función de Dulac $d$}

En una vecindad de la silla $\left(1, \sqrt{\frac{1}{-F}}\right)$, el sistema Loud es analíticamente equivalente a una ecuación diferencial lineal. En efecto, la función

$$
\begin{aligned}
\phi:] \frac{1}{2 F},+\infty\left[\times \mathbf{R}^{+}\right. & \rightarrow \Delta_{2}=\left\{(u, v) \in \mathbf{R}^{2}: u<1-\frac{1}{2 F}, v<-\frac{u}{1-2 F}-\frac{1}{2 F}\right\} \\
(x, y) & \mapsto \phi(x, y)=(u, v)=\left(1-x,-\frac{y^{2}}{2}-\frac{(1-x)}{1-2 F}-\frac{1}{2 F}\right)
\end{aligned}
$$

es un difeomorfismo analítico, cuya inversa es la función definida por

$$
\phi^{-1}(u, v)=\left(1-u, \sqrt{-2 v-\frac{2 u}{1-2 F}-\frac{1}{F}}\right)=(x, y) .
$$

La función $\phi$ lleva la silla $\left(1, \sqrt{\frac{1}{-F}}\right)$ al origen del sistema $(u, v)$ y transforma el sistema Loud en

$$
\left\{\begin{array}{l}
\frac{d u}{d t}=\sqrt{-2 v-\frac{2 u}{1-2 F}-\frac{1}{F}} u \\
\frac{d v}{d t}=\sqrt{-2 v-\frac{2 u}{1-2 F}-\frac{1}{F}} 2 F v .
\end{array}\right.
$$

Así, el sistema Loud es analíticamente equivalente a

$$
\left\{\begin{array}{l}
\frac{d u}{d t}=u \\
\frac{d v}{d t}=2 F v .
\end{array}\right.
$$

Consideremos las siguientes secciones transversales del sistema 2.2:

$$
\left.\bar{\sigma}_{1}:\right]-\delta_{1}, \delta_{1}\left[\rightarrow \Delta_{2}, u_{0} \mapsto \bar{\sigma}_{1}\left(u_{0}\right)=\left(u_{0}, 1\right) \quad \text { y } \quad \bar{\pi}_{1}:\right]-\epsilon_{1}, \epsilon_{1}\left[\rightarrow \Delta_{2}, v_{0} \mapsto \bar{\pi}_{1}\left(v_{0}\right)=\left(1, v_{0}\right)\right.
$$


Notemos que para $\delta_{1}>0$ y $\epsilon_{1}>0$ suficientemente pequeños los segmentos $\bar{\Sigma}_{1}=\bar{\sigma}_{1}(]-\delta_{1}, \delta_{1}[)$ y $\bar{\Pi}_{1}=\bar{\pi}_{1}(]-\epsilon_{1}, \epsilon_{1}[)$ están contenidos en $\Delta_{2}$.

La secciones transversales $\sigma_{1}$ y $\pi_{1}$ que tomaremos (mencionadas al comienzo de esta sección) son $\sigma_{1}=\phi^{-1} \circ \bar{\sigma}_{1}$ y $\pi_{1}=\phi^{-1} \circ \bar{\pi}_{1}$.

La curva integral del sistema 2.2 que pasa por $\left(u_{0}, 1\right)$, con $u_{0}>0$, corta a la transversal $\bar{\Pi}_{1}$ en el punto $\left(1, d\left(u_{0}\right)\right)=\left(1, u_{0}^{-2 F}\right)$. Tenemos:

Proposition 2.1. La función de Dulac $d:] 0, \delta_{1}[\times]-\frac{1}{2} ; 0[\rightarrow] 0, \epsilon_{1}[$ está definida por

$$
\left(u_{0} ; F\right) \mapsto d\left(u_{0} ; F\right)=u_{0}^{-2 F} \text {. }
$$

\subsection{Estudio de la función $f_{1}$.}

Consideremos el cambio de coordenadas siguiente:

$$
\psi:(x, y) \mapsto(w, z)=(y, 1-x) .
$$

En las coordenadas $(w, z)$ el sistema Loud se escribe como

$$
X_{F}:\left\{\begin{array}{l}
\frac{d w}{d t}=-z+1+F w^{2}=f(w, z) \\
\frac{d z}{d t}=z w=z g(w, z)
\end{array}\right.
$$

Las funciones $\xi=\psi \circ \sigma$ y $\eta=\psi \circ \sigma_{1}$, donde $\sigma_{1}$ es la parametrización de $\Sigma_{1}$ introducida en la sección anterior, son secciones transversales de 2.3 y están definidas por

$$
\begin{aligned}
\xi:]-\delta, \delta[ & \rightarrow \mathbf{R}^{2} \\
s & \mapsto \xi(s)=(0, s)
\end{aligned}
$$

y

$$
\begin{aligned}
\eta:]-\delta_{1}, \delta_{1}[ & \rightarrow \mathbf{R}^{2} \\
u_{0} & \mapsto \eta\left(u_{0}\right)=\left(\sqrt{-\frac{2 u_{0}}{1-2 F}-\frac{1}{F}-2}, u_{0}\right) .
\end{aligned}
$$

De [3], Lema 3.2, existe una función $h_{1}$, analítica en una vecindad de $s=0$ y $F \in]-1 / 2,0[$, tal que

$$
f_{1}(s ; F)=\Delta_{1}(F) s+s h_{1}(s ; F),
$$

con

$$
\Delta_{1}(F)=\frac{\xi_{2}^{\prime}(0)}{\eta_{2}^{\prime}(0)} \cdot \exp \left(\int_{\xi_{1}(0)}^{\eta_{1}(0)} \frac{g(w, 0 ; F)}{f(w, 0 ; F)} d w\right)
$$


donde $\left(\xi_{1}, \xi_{2}\right)$ y $\left(\eta_{1}, \eta_{2}\right)$ son las coordenadas de $\xi$ y $\eta$, respectivamente. En nuestro caso

$\Delta_{1}(F)=\exp \left(\int_{0}^{\sqrt{-\frac{1}{F}-2}} \frac{w}{1+F w^{2}} d w\right)=\exp \left(\frac{1}{2 F}(\ln (-2 F)-\ln 1)\right)=(-2 F)^{\frac{1}{2 F}}$

y obtenemos la siguiente proposición, considerando un $\delta$ más pequeño, si es necesario:

Proposition 2.2. Existe una función analítica $\left.h_{1}:\right]-\delta, \delta[\times]-1 / 2,0[\rightarrow$ $\mathbf{R}^{+},(s ; F) \mapsto h_{1}(s ; F)$ tal que para $\left.s \in\right] 0, \delta[$,

$$
f_{1}(s ; F)=(-2 F)^{\frac{1}{2 F}} s\left(1+h_{1}(s ; F)\right)
$$

con $h_{1}(0 ; F)=0$, para todo $\left.F \in\right]-1 / 2,0[$.

\subsection{Estudio de la función $f_{2}$.}

En esta sección demostraremos la siguiente proposición:

Proposition 2.3. Existe $\epsilon_{1}>0$ tal que la función de Poincaré $f_{2}$ verifica

$$
f_{2}\left(v_{0} ; F\right)=\frac{(1-2 F)^{1+2 F}}{(-2 F)^{2 F}} v_{0}\left(1+h_{2}\left(v_{0} ; F\right)\right),
$$

con $h_{2}$ una función analítica en $]-\epsilon_{1}, \epsilon_{1}[\times]-1 / 2,0\left[\right.$ y $h_{2}(0 ; F)=0$ para todo $F \in]-1 / 2,0[$.

Proof. Realizaremos un cambio de coordenadas de manera que los puntos de la parábola con segunda coordenada positiva estén, en las nuevas coordenadas, sobre el eje horizontal.

Sea

$$
\begin{aligned}
\varphi: \mathbf{R}^{-} \times \mathbf{R}^{+} & \rightarrow \Delta_{3} \\
(x, y) & \mapsto \varphi(x, y)=\left(-x,-\frac{y^{2}}{2}-\frac{1-x}{1-2 F}-\frac{1}{2 F}\right)=(u, v),
\end{aligned}
$$

donde

$$
\Delta_{3}=\left\{(u, v): u>0, v<\frac{1+u}{1-2 F}-\frac{1}{2 F}\right\}
$$


La función $\varphi$ es un difeomorfismo analítico cuya inversa es la función

$$
\begin{aligned}
\varphi^{-1}: \Delta_{3} & \rightarrow \mathbf{R}^{-} \times \mathbf{R}^{+} \\
(u, v) & \mapsto \varphi^{-1}(u, v)=\left(-u, \sqrt{-2 v-\frac{2(1+u)}{1-2 F}-\frac{1}{F}}\right)=(x, y) .
\end{aligned}
$$

Se tiene que $\varphi\left(0, \sqrt{\frac{1}{-F(1-2 F)}}\right)=(0,0)$. Así, el origen del sistema de coordenadas $(u, v)$ está en el punto $\left(0, \sqrt{\frac{1}{-F(1-2 F)}}\right)$, que corresponde al punto de intersección de la parábola $\frac{y^{2}}{2}+\frac{1-x}{1-2 F}+\frac{1}{2 F}=0$ con el semieje $y$ positivo. El eje $u$ corresponde a la curva $\left\{(x, y):-\frac{y^{2}}{2}-\frac{1-x}{1-2 F}-\frac{1}{2 F}=0, y>0\right\}$.

En las coordenadas $(u, v)$, el sistema Loud se escribe como

$$
\left\{\begin{array}{l}
\frac{d u}{d t}=\sqrt{-2 v-\frac{2(1+u)}{1-2 F}-\frac{1}{F}}(1+u) \\
\frac{d v}{d t}=\sqrt{-2 v-\frac{2(1+u)}{1-2 F}-\frac{1}{F}} 2 F v .
\end{array}\right.
$$

Haciendo un reescalamiento en el tiempo, obtenemos

$$
\left\{\begin{array}{l}
\frac{d u}{d t}=1+u \\
\frac{d v}{d t}=2 F v
\end{array}\right.
$$

Observemos que la transversal $\Pi$ no está contenida en el dominio de $\varphi$, debido a que $\varphi$ no es un difeomorfismo en una vecindad del vértice $\left(\frac{1}{2 F}, 0\right)$ de la parábola, por lo que vamos a escoger una transversal cercana a $\Pi$.

Sea $(a(\beta), \beta)$ un punto de la parábola $\frac{y^{2}}{2}+\frac{1-x}{1-2 F}+\frac{1}{2 F}=0$, con $\beta>0$ suficientemente pequeño. Consideremos la transversal $\Pi_{\beta}$ parametrizada por:

$$
\begin{aligned}
\left.\pi_{\beta}:\right]-1,1[ & \rightarrow \mathbf{R}^{2} \\
s_{0} & \mapsto \pi_{\beta}\left(s_{0}\right)=\left(a(\beta)+s_{0}, \beta\right) .
\end{aligned}
$$

Observemos que $a(\beta)=\frac{(1-2 F) \beta^{2}}{2}+\frac{1}{2 F}$, y si $\beta \rightarrow 0^{+}$entonces $a(\beta) \rightarrow \frac{1}{2 F}$. Así, $(a(\beta), \beta)$ converge a $\left(\frac{1}{2 F}, 0\right)$, cuando $\beta \rightarrow 0^{+}$.

Consideremos los segmentos de recta transversales al sistema $2.5, \widetilde{\Pi}_{1}$ y $\widetilde{\Pi}_{\beta}$, parametrizados respectivamente por las funciones $\varphi \circ \pi_{1}$ y $\varphi \circ \pi_{\beta}$, definidas respectivamente por: 


$$
\begin{aligned}
\left.\varphi \circ \pi_{1}:\right]-\epsilon_{1}, \epsilon_{1}[ & \rightarrow \mathbf{R}^{2} \\
v_{0} & \mapsto \varphi \circ \pi_{1}\left(v_{0}\right)=\left(0, v_{0}\right)
\end{aligned}
$$

$\mathrm{y}$

$$
\begin{aligned}
\left.\varphi \circ \pi_{\beta}:\right]-1,1[ & \rightarrow \mathbf{R}^{2} \\
s_{0} & \mapsto \varphi \circ \pi_{\beta}\left(s_{0}\right)=\left(-a(\beta)-s_{0}, \frac{a(\beta)+s_{0}}{1-2 F}-\frac{\beta^{2}}{2}-\frac{1}{2 F(1-2 F)}\right) .
\end{aligned}
$$

Para el punto intersección $(u, v)$ de esta órbita con el segmento $\widetilde{\Pi}_{\beta}$, existe $\left.s_{0} \in\right]-1,1\left[\right.$ tal que $u=-a(\beta)-s_{0}$ y así

$$
v=v_{0}(1+u)^{2 F}=\frac{a(\beta)+s_{0}}{1-2 F}-\frac{\beta^{2}}{2}-\frac{1}{2 F(1-2 F)} .
$$

Es decir,

$$
v_{0}=\left(1-a(\beta)-s_{0}\right)^{-2 F}\left(\frac{a(\beta)+s_{0}}{1-2 F}-\frac{\beta^{2}}{2}-\frac{1}{2 F(1-2 F)}\right) .
$$

Haciendo tender $\beta \rightarrow 0^{+}$, se obtiene

$$
v_{0}=\frac{(1-2 F)^{-1-2 F}}{(-2 F)^{-2 F}} s_{0}\left(1+\frac{2 F}{1-2 F} s_{0}\right)^{-2 F} .
$$

Esta igualdad define, para $s_{0}$ suficientemente pequeño, a la inversa de $f_{2}$. Por el teorema de la función inversa existe $\epsilon_{1}>0$ y una función $h_{2}$ analítica en $]-\epsilon_{1}, \epsilon_{1}[\times]-\frac{1}{2}, 0\left[\operatorname{con} h_{2}(0 ; F)=0\right.$, para todo $\left.F \in\right]-\frac{1}{2}, 0[$, tal que

$$
f_{2}\left(v_{0} ; F\right)=\frac{(1-2 F)^{1+2 F}}{(-2 F)^{2 F}} v_{0}\left(1+h_{2}\left(v_{0} ; F\right)\right) \text {. }
$$

Lo que demuestra la Proposición.

\subsection{Finalización de la demostración del Teorema A}

Recordemos que la función $R$ es la compuesta de las funciones $f_{2}, d$ y $f_{1}$ estudiadas en las secciones anteriores, es decir, $R=f_{2} \circ d \circ f_{1}$. La función $d$ es del tipo $x^{\lambda}$ y las funciones $f_{1}$ y $f_{2}$ son analíticas en cero, en particular ellas pertenecen al grupo de Dulac. Así, la compuesta $R$ es una función de Dulac.

Tenemos, 
$v_{0}=d\left(f_{1}(s ; F)\right)=(-2 F)^{\frac{-2 F}{2 F}} s^{-2 F}\left(1+h_{1}(s ; F)\right)^{-2 F}=-\frac{1}{2 F} s^{-2 F}\left(1+h_{1}(s ; F)\right)^{-2 F}$.

Así, finalmente, se obtiene

$$
R(s ; F)=f_{2}\left(d\left(f_{1}(s ; F)\right)\right)=\left(\frac{1-2 F}{-2 F}\right)^{1+2 F} s^{-2 F}\left(1+r_{1}(s ; F)\right),
$$

con $1+r_{1}(s ; F)=\left(1+h_{1}(s ; F)\right)^{-2 F}\left(1+h_{2}\left(f_{1}(s ; F) ; F\right)\right)$ con $\lim _{s \rightarrow 0^{+}} r_{1}(s ; F)=$ 0 . Asíqueda demostrado el teorema.

\section{References}

[1] C. Chicone, review in MathScinet, ref. 94h:58072.

[2] Dulac, H. Sur les cycles limites. Bull. Soc. Math. France 51, pp. 45-188, (1923).

[3] Mardešić, P, Marín, D and Villadelprat, J. On the time function of the Dulac map for families of meromorphic vector fields, Nonlinearity, 16, pp. 855-881, (2003).

[4] Mardešić, P, Marín, D and Villadelprat, J. The period function of reversible quadratic centers, J. Differential Equations 224, pp. 120171, (2006).

[5] Moussu, R. Le problème de la finitude du nombre de cycles limites, Séminaire Bourbaki, exp. N 655, pp. 89-101, (1985).

\section{Nicole Carrasco Vidal}

Departamento de Matemática,

Facultad de Ciencias Físicas y Matemáticas

Universidad de Concepción, Chile

e-mail: nicolecarrasco@udec.cl 